\title{
Quasicrystal metamaterials: a route to optical isotropy
}

\author{
S. Kruk ${ }^{1}$, C. Helgert ${ }^{1,2}$, M. Decker ${ }^{1}$, I. Staude ${ }^{1}$, C. Etrich ${ }^{2}$, C. Menzel ${ }^{2}$, C. Rockstuhl' \\ C. Jagadish ${ }^{3}$, T. Pertsch ${ }^{2}$, D. N. Neshev ${ }^{1}$, and Yu. S. Kivshar ${ }^{1}$ \\ 1. Nonlinear Physics Centre and Centre for Ultrahigh-bandwidth Devices for Optical Systems (CUDOS), \\ Research School of Physics and Engineering, The Australian National University, Canberra, ACT 0200, Australia \\ 2. Institute of Applied Physics, Abbe Center of Photonics, Friedrich-Schiller-Universität Jena, 07743 Jena, Germany \\ 3. Department of Electronic Materials and Engineering, \\ Research School of Physics and Engineering, The Australian National University, Canberra, ACT 0200, Australia \\ sergey.kruk@anu.edu.au
}

\begin{abstract}
We introduce a novel class of metamaterials with quasicrystalline meta-atom arrangements and study their properties in comparison with periodic and disordered metamaterials. We show that quasicrystalline metamaterials exhibit isotropic optical properties while preserving pronounced resonances.

OCIS codes: (160.3918) Metamaterials; (260.5430) Polarization; (310.6628) Subwavelength structures, nanostructures.
\end{abstract}

\section{Introduction}

The concept of metamaterials offers exceptional opportunities for creating artificial materials with virtually unlimited range of macroscopic properties, including such that are remarkably different from natural materials [1]. Optical metamaterials rely on the ability to fabricate nanoscale metal-dielectric particles of various shapes (meta-atoms) arranged in a specific pattern. This approach allows us to create at will composite metamaterials optimized for a desired operation and functionality. In recent years, it became evident that interactions between the single meta-atoms in the array play an important role for the metamaterial response [2]. Thus, the inherent arrangement of identical metal-dielectric nanoparticles affects the macroscopic optical properties of metamaterials. However, up to date most of the studies of various properties of metamaterials are based on a silent assumption that, similar to most solids in nature, the meta-atoms are composed in a periodic lattice. One of the main disadvantages of such an arrangement is the anisotropic response of the metamaterial for the case of oblique incidence of light. In order to obtain isotropic optical response, metamaterials with disordered arrangement of elements have been proposed [3, 4]. However, disorder increases scattering of the light dramatically, which reduces the resonant power of the structure.

In this letter we introduce a novel class of metamaterials with a quasicrystalline meta-atom arrangement. Such an arrangement has long-range positional order, but no periodicity. Therefore quasicrystalline metamaterials exhibit isotropic optical response in contrast to periodic metamaterials while preserving same level of scattering. The effect of the meta-atom interactions is measured by examining the extrinsic optical activity $[5,6]$ of the different metamaterials at the oblique incidence, which allow us to be only sensitive to the optical properties induced by the symmetry characteristics of the underlying lattice. As such, we reveal that qualitatively different optical properties of metamaterials can be achieved by controlling the geometry of their nanoparticle constituents and predict a novel type of metamaterials, quasicrystalline metamaterials.

\section{Symmetry-dependent optical properties of metamaterials}

In our experiments we utilize a magnetic optical metamaterial composed of meta-atoms consisting of two Au disks each $25 \mathrm{~nm}$ thin, separated by a $30 \mathrm{~nm} \mathrm{MgF}$ layer (Fig. 1a). The meta-atom has a circular symmetry with bottom diameter of $180 \mathrm{~nm}$ and weak tapering of $10^{\circ}$. The identical meta-atoms are placed on a glass substrate and arranged into three types of symmetries: periodic (and consequently ordered) [Fig. 1(b)], quasicrystalline (ordered, but non-periodic) [Fig. 1(c)], and disordered non-periodic array [Fig. 1(d)]. By comparing the properties of those three samples we can independently study the impact of both order and periodicity and emphasize the influence of the unique quasicrystalline arrangement.
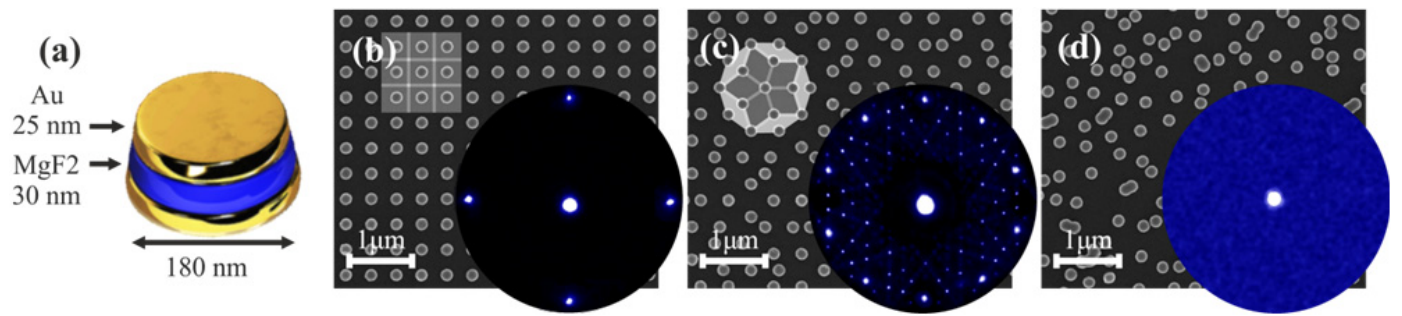

Fig. 1. (a) A single metamaterial magnetic atom; and scanning electron micrographs of (b) periodic, (c) quasicrystalline, and (d) disordered metamaterials. Inserts show the far-field diffraction patterns of the metamaterials obtained with a laser beam of $0.4 \mu \mathrm{m}$ wavelength. The diffraction pattern of the quasicrystalline metamaterial shows the lack of long-range positional order, while the microscopic image of the elements show the absence of periodicity. 
The impact of the order of meta-atom arrangement can be directly observed by comparing the strength of the resonances of our metamaterials. To precisely measure the impact of periodicity of meta-atoms arrangements we study the extrinsic optical chirality of the metamaterials. The extrinsic chirality of internally non-chiral object appears when the direction of propagation of light isn't parallel to any of the mirror planes of the object. In our case the single meta-atom is a disk, i.e. it has infinite number of mirror planes, therefore it cannot exhibit chirality by itself. However, the arrangement of the meta-atoms may still have a finite number of mirror planes. Therefore, by measuring chirality of our metamaterial, we observe the impact of the meta-atom arrangement only, regardless the impact of a single meta-atom.

To characterize chirality of the metamaterials we study the transmission of circular-polarized light at oblique incidence onto the sample. Fig. 2 shows the experimental setup and an example of mutual orientation of the light wave and the metamaterial, which gives rise to extrinsic chirality.
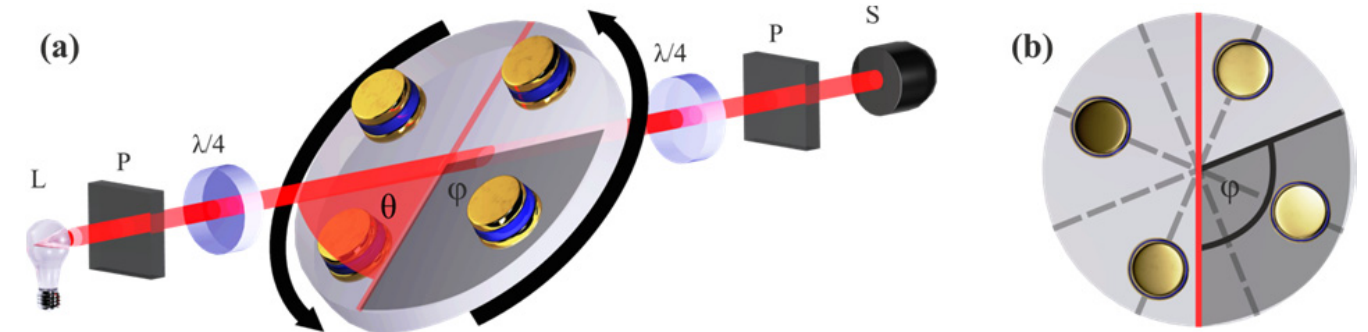

Fig. 2. (a) Experimental setup: white light source L, polarizers $\mathrm{P}$, achromatic quarter-wave plates $\lambda / 4$, and spectrometer S. Angle $\theta$ is measured in the plane of incidence of light, angle $\phi$ is measured in the sample plane. (b) Top view on the mirroring planes of the periodic metamaterial (dashed gray) with respect to the plane of incidence of light (red).

Top plots in Fig. 3 shows the transmission spectra of left-circularly polarized light for $\theta=45^{\circ}$. Radial coordinates of each polar plot correspond to different wavelengths in the range 0.5-1.0 $\mu \mathrm{m}$; angular coordinates correspond to different $\phi$ angles. Next we examine chirality by calculating circular dichroism as the difference of amplitudes of transmitted left- and right-circularly polarized light: $\left[L C P_{a m p}-R C P_{a m p}\right] /\left[L C P_{a m p}+R C P_{a m p}\right]$, as shown in the bottom plots of Fig. 3. We observe that all three transmission spectra have two resonant lines: near $0.7 \mu \mathrm{m}$ (electric dipole resonance) and near $0.95 \mu \mathrm{m}$ (magnetic dipole resonance). We notice that the spectra of the metamaterials with ordered lattices are much sharper in comparison with the disordered metamaterial. We also observe that periodic metamaterial exhibits strong circular dichroism and therefore chirality for angles $\phi \neq \pi / 4$, which is a result of interaction induced anisotropy. As one could expect, the disordered metamaterials shows no chirality. Most importantly, the quasicrystalline metamaterial shows no chirality and therefore strong isotropy.
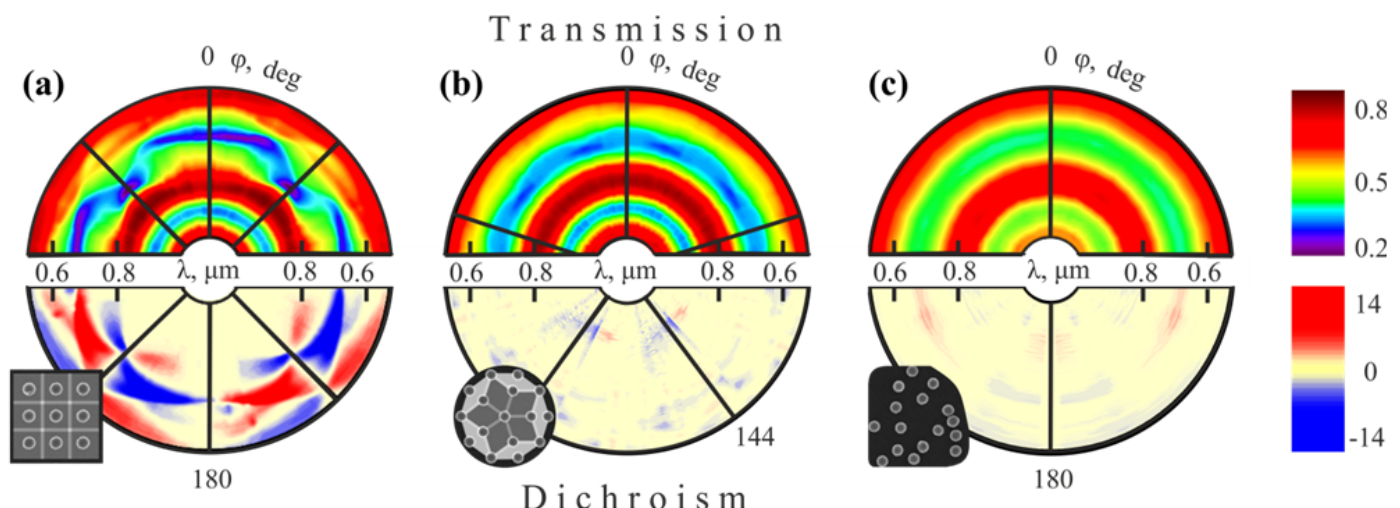

Fig. 3. Transmission of left-circularly polarized light (top plots) and circular dichroism (bottom plots) of (a) periodic metamaterial, (b) quasicrystalline metamaterial and (c) disordered metamaterial. Radial coordinates of each polar plot correspond to different wavelengths, angular coordinates correspond to different $\phi$ angles.

In conclusion, we have introduced the concept of quasicrystalline metamaterials and revealed that their unique symmetry leads to optical isotropy while preserving the resonance strength of the metamaterial.

\section{References}

1. C. M. Soukoulis and M. Wegener, "Past achievements and future challenges in the development of three-dimensional photonic metamaterials," Nat. Photon. 5, 523-530 (2011).

2. M. Decker, S. Linden, and M. Wegener, "Coupling effects in low-symmetry planar split-ring resonator arrays," Opt. Lett. 34, 1579 (2009).

3. C. Helgert, C. Rockstuhl, C. Etrich, C. Menzel, E. B. Kley, A. Tünnermann, F. Lederer, and T. Pertsch, "Effective properties of amorphous metamaterials," Phys. Rev. B 79, 233107 (2009).

4. N. Papasimakis, et. al "Coherent and incoherent metamaterials and order-disorder transitions," Phys. Rev. B 80, 041102 (2009).

5. M. Born and W. E., Principles of Optics (Pergamon Press, 1959).

6. E. Plum, V. A. Fedotov, and N. I. Zheludev, "Optical activity in extrinsically chiral metamaterial," Appl. Phys. Lett. 93, 191911 (2008). 